\title{
Neurosyphilis presenting as acute ischemic stroke
}

\author{
Authors: Dhayalen Krishnan, ${ }^{A}$ Siti SM Zaini, ${ }^{B}$ Kartikasalwah A Latif ${ }^{C}$ and Joyce P Joseph ${ }^{D}$
}

\begin{abstract}
Neurosyphilis is a broad term used to describe an infection caused by Treponema pallidum in the central nervous system. While this was a common cause of stroke in the 19th century, it saw a decline after the introduction of penicillin. However, in the recent past, there has been an increase in the incidence of syphilis, especially with HIV coinfection. Neurosyphilis results from an untreated primary syphilis. Neuropsychiatric disorder appears to be the commonest manifestation followed cerebrovascular accident, myelopathy, ocular disease and seizure. Known as the 'great imitator', this entity, however, may be easily missed if not for a high index of suspicion. This is especially so because of its similar presentation to other more common clinical conditions. We describe the case of a 39-year-old man displaying acute global aphasia and right-sided facial weakness in keeping with a left middle cerebral artery infarct. This was confirmed with computed tomography of the brain, and subsequently, further investigations revealed a diagnosis of neurosyphilis. The patient was treated with intravenous benzylpenicillin and recovered well with treatment.
\end{abstract}

KEYWORDS: Meningovascular syphilis, stroke, arteritis, MRI

\section{Introduction}

Syphilis is a common sexually transmitted disease caused by the spirochete Treponema pallidum. Illicit drug usage, such as cocaine and methamphetamine, and men who have sex with men (MSM) were identified as the main risk factors to contract this disease. ${ }^{1,2}$ Once acquired, and if untreated, the disease progresses into four stages; namely latent, primary, secondary and tertiary, with neurosyphilis being a form of tertiary syphilis. Neurosyphilis is commonly seen in HIV infected individuals. ${ }^{1}$ The diagnosis of neurosyphilis still remains a challenge, especially in patients without a known syphilis infection as the disease can present in atypical forms. Neurological symptoms with little or no evidence of primary or secondary syphilis may well be the first manifestation.

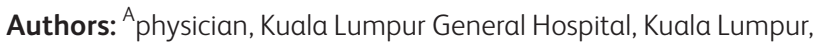

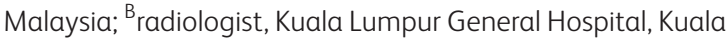
Lumpur, Malaysia; ' neurologist, Kuala Lumpur General Hospital, Kuala Lumpur, Malaysia; ${ }^{D}$ neuroradiologist, Kuala Lumpur General Hospital, Kuala Lumpur, Malaysia
However, prognosis remains good, provided the disease is detected early and appropriate treatment is promptly initiated.

\section{Case presentation}

A 39-year-old man was brought to the hospital as a result of a sudden onset of speech difficulty. He had been experiencing a mild dull constant headache for the past week. On the night preceding admission, he was noted to have had several episodes of transient right facial asymmetry, each lasting less than a minute. There was no fever, behavioural change nor limb weakness, and consciousness remained intact. Clinical examination revealed a thin gentleman who had global aphasia. He had right upper motor neuron facial weakness. He could move all four limbs, and no drifting was noted on lifting the limbs above gravity. Multiple mildly enlarged cervical lymph nodes were palpable bilaterally. Physical examination, including his blood pressure, was otherwise unremarkable.

Computed tomography of the brain showed a left middle cerebral artery (MCA) territory infarct with thrombosis at the M1 segment of the left MCA. Magnetic resonance imaging done on the brain later confirmed the left MCA region infarct (Fig 1) with significant M1 segment stenosis. Additionally, the left M1, M2 and $\mathrm{M} 3$ branches of the MCA and left anterior cerebral artery appeared beaded and showed vascular wall enhancement with contrast (Fig 2). Leptomeningeal enhancement was seen around the infarcted region (Fig 3). Preliminary blood investigations revealed lymphopenia $0.6 \times 10^{3}$ cells/L (1-3.7). He was investigated further, on noting that he did not have any conventional cardiovascular risk factors. His serum HIV, Venereal Disease Research Laboratory (VDRL) test and Treponema pallidum particle agglutination assay (TPPA) test were positive with VDRL titre of 1:256. This prompted us to perform a lumbar puncture, which revealed cerebrospinal fluid (CSF) that was reactive for VDRL with a titre of 1:8. There were no CSF pleocytosis, and all other CSF parameters were within normal limits. This confirmed a diagnosis of neurosyphilis, and the patient was commenced on intravenous (IV) benzylpenicillin as per protocol.

\section{Discussion}

Syphilis is a sexually transmitted disease caused by the spirochete Treponema pallidum for which humans are the only host. There is a worldwide re-emergence of this infection especially in urban areas and more so among HIV-infected individuals. ${ }^{3-6}$ Early involvement of the central nervous system is seen in patients with untreated primary or secondary syphilis. 
Fig 1. Isotropic trace diffusionweighted imaging (left) and apparent diffusion coefficient (right) maps show restricted diffusion in left lentiform nucleus and left caudate nucleus representing acute left middle cerebral artery vascular territory infarcts.

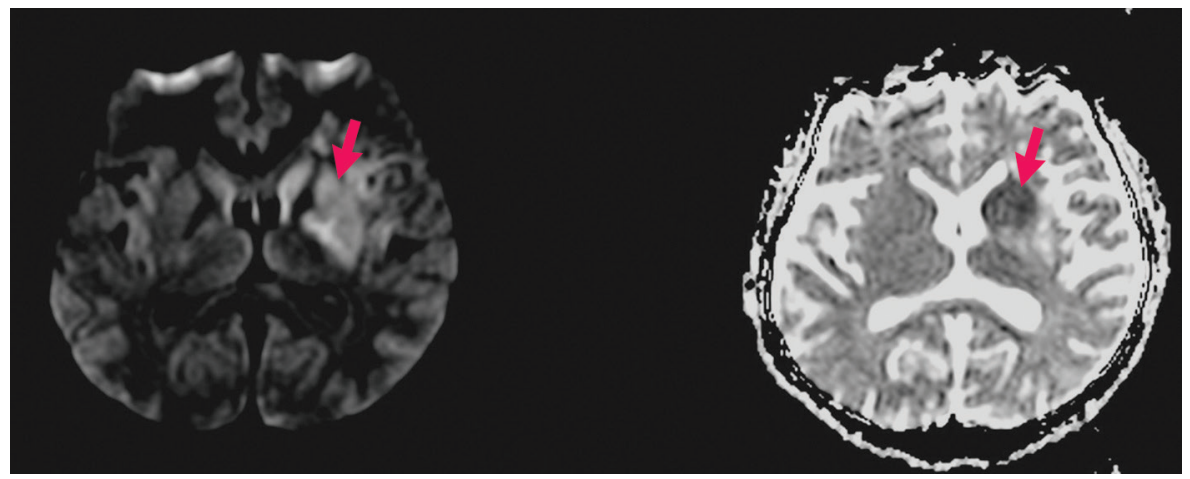

Clinical manifestations of neurosyphilis may vary greatly from asymptomatic to neuropsychiatric infections, meningovascular or myelopathic disorders; this makes it a great mimicker of several diseases. ${ }^{6}$ Meningovascular syphilis is caused by obliterative endarteritis involving the medium (Heubner's) and small-sized (Nissl-Alzheimer) intracranial vessels. The resulting inflammation with fibroblastic and collagenous proliferation within the vessel walls leads to luminal narrowing, cerebrovascular thrombosis and ischaemic infarction. ${ }^{7}$ A stroke in the distribution of MCA territory is the most common presentation followed by an involvement of the basilar artery and its branches. Prodromal symptoms such as headache, dizziness or behavioural changes are noted in the preceding days or weeks. In the right clinical context, a positive CSF VDRL test is diagnostic of neurosyphilis. CSF VDRL has high specificity of up to $90 \%$ but low sensitivity. ${ }^{8}$ There is also a possibility of false positive results if the CSF sample is

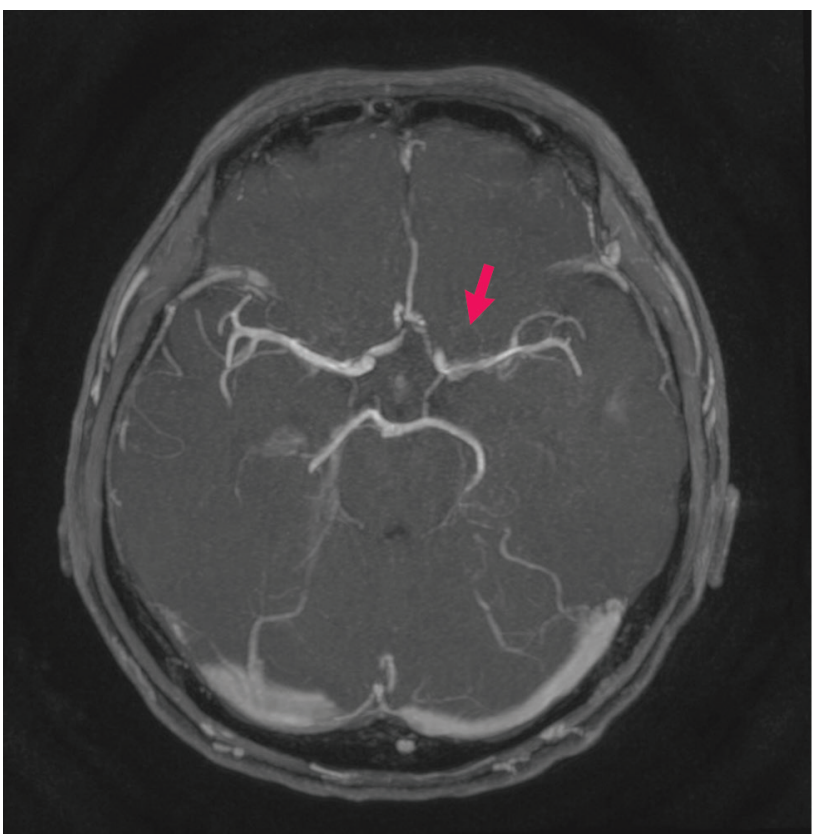

Fig 2. Contrast-enhanced magnetic resonance imaging of the brain showing short segment severe stenosis at M1 segment of left middle cerebral artery (red arrow) with irregular, thickened enhancing vessel wall. contaminated with a substantial amount of blood, especially if the serum VDRL titres are low. ${ }^{9,10}$ Classically, CSF pleocytosis and elevated protein are expected in neurosyphilis; however, normal findings have been reported as well. ${ }^{11}$

Our patient displayed an acute ischaemic stroke involving the left MCA territory, with mild prodromal symptoms of headache. However, he was not eligible for the timely interventions such as IV recombinant tissue plasminogen activator therapy or thrombectomy due to delay in presentation. A secondary aetiology was suspected based on the constellation of additional findings such as multiple cervical lymphadenopathy and lymphopenia on repeated complete blood counts. He was treated with IV benzylpenicillin after which he showed good recovery. This case highlights the need for a high index of suspicion for meningovascular syphilis in patients who present with acute ischaemic stroke with minimal or no significant risk factors. .

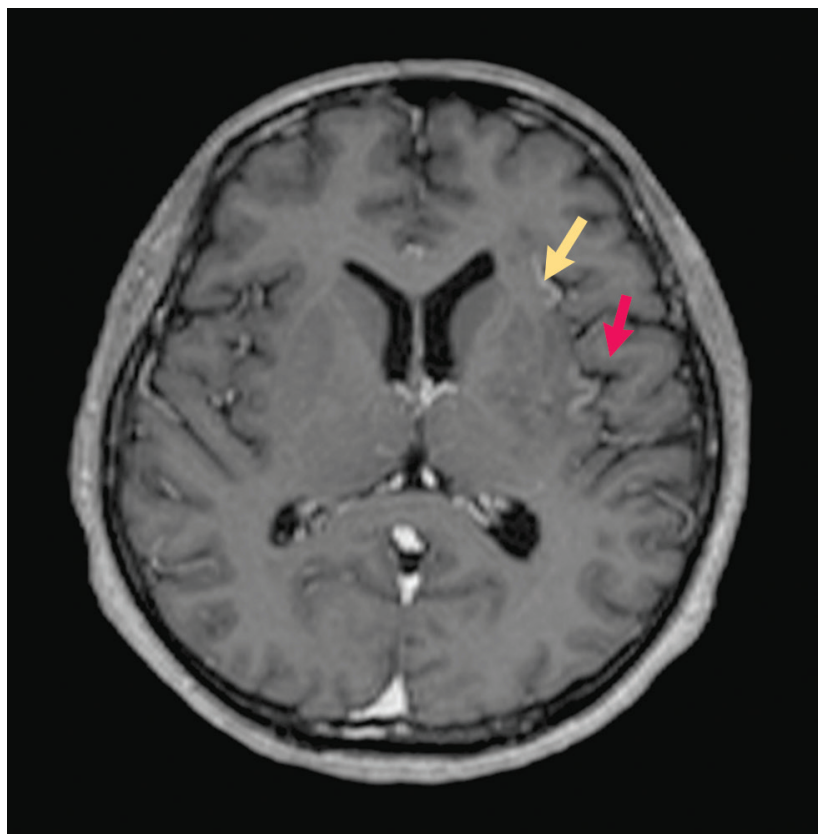

Fig 3. Contrast-enhanced magnetic resonance imaging of the brain showing cortical enhancement at left insular (red arrow) and patchy enhancement at left basal ganglia at the areas of infarct (yellow arrow). 


\section{References}

1 Wong W, Chaw JK, Kent CK, Klausner JD. Risk factors for early syphilis among gay and bisexual men seen in an STD clinic: San Francisco, 2002-2003. Sex Transm Dis 2005;32:458-63.

2 Golden MR, Marra CM, Holmes KK. Update on syphilis: resurgence of an old problem. JAMA 2003;290:1510-4.

3 Mattei PL, Beachkofsky TM, Gilson RT, Wisco O]. Syphilis: a reemerging infection. Am Fam Physician 2012;86:433-40.

4 Chahine LM, Khoriaty RN, Tomford WJ, Hussain MS. The changing face of neurosyphilis. Int ] Stroke 2011;6:136-43.

5 Lee JP, Koo SH, Jin SY, Kim TH. Experience of meningovascular syphilis in human immunodeficiency virus infected patient. J Korean Neurosurg Soc 2009;46:413-6.

6 Hobbs E, Vera JH, Marks M et al. Neurosyphilis in patients with HIV. Practical Neurology 2018;18:211-8.

7 Pezzini A, Gulletta M, Pinelli L et al. Meningovascular syphilis: A vascular syndrome with typical features? Cerebrovasc Dis 2001;11:352-3.
8 Zhu L, Gu X, Peng RR et al. Comparison of the cerebrospinal fluid (CSF) toluidine red unheated serum test and the CSF rapid plasma reagin test with the CSF venereal disease research laboratory test for diagnosis of neurosyphilis among HIV-negative syphilis patients in China. J Clin Microbiol 2014;52:736-40.

9 Abkur TM, Ahmed GS, Alfaki NO et al. Neurosyphilis presenting with a stroke-like syndrome. BMJ Case Rep 2015;2015:bcr2014206988.

10 Izzat NN, Bartruff JK, Glicksman JM et al. Validity of the VDRL test on cerebrospinal fluid contaminated by blood. $\mathrm{Br}]$ Vener Dis 1971:47:162-4.

11 Marra CM, Critchlow CW, Hook EW 3rd, Collier AC, Lukehart SA. Cerebrospinal fluid treponemal antibodies in untreated early syphilis. Arch Neurol 1995;52:68-72.

Address for correspondence: Dr Dhayalen Krishnan, Department of Neurology, Kuala Lumpur General Hospital, Jalan Pahang, 50586 Kuala Lumpur, Malaysia.

Email:kdhayalen@yahoo.com

\section{Royal College} of Physicians

\section{Guidance on safe medical staffing}

The RCP's Guidance on safe medical staffing working party report aims to help those planning and organising core hospital medical services to answer the question: 'How many doctors or their alternatives, with what capabilities, do we need to provide safe, timely and effective care for patients with medical problems?'.

\section{Download the report at: www.rcplondon.ac.uk/safe-medical-staffing}
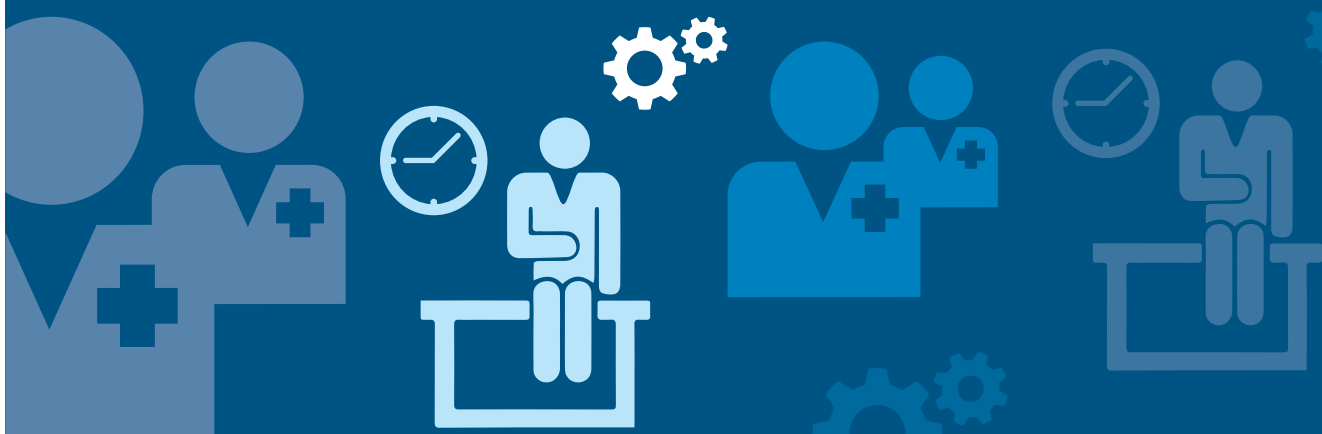